\title{
Transfer of children with isolated linear skull fractures: is it worth the cost?
}

\author{
Ian K. White, MD, ${ }^{1}$ Ecaterina Pestereva, BS, ${ }^{1}$ Kashif A. Shaikh, MD, ${ }^{1}$ and Daniel H. Fulkerson, MD $^{2}$ \\ 1Department of Neurological Surgery, Indiana University School of Medicine; and 2Department of Neurological Surgery, Division \\ of Pediatric Neurosurgery, Goodman Campbell Brain and Spine, Indiana University School of Medicine, Indianapolis, Indiana
}

\begin{abstract}
OBJECTIVE Children with skull fractures are often transferred to hospitals with pediatric neurosurgical capabilities. Historical data suggest that a small percentage of patients with an isolated skull fracture will clinically decline. However, recent papers have suggested that the risk of decline in certain patients is low. There are few data regarding the financial costs associated with transporting patients at low risk for requiring specialty care. In this study, the clinical outcomes and financial costs of transferring of a population of children with isolated skull fractures to a Level 1 pediatric trauma center over a 9-year period were analyzed.
\end{abstract}

METHODS A retrospective review of all children treated for head injury at Riley Hospital for Children (Indianapolis, Indiana) between 2005 and 2013 was performed. Patients with a skull fracture were identified based on ICD-9 codes. Patients with intracranial hematoma, brain parenchymal injury, or multisystem trauma were excluded. Children transferred to Riley Hospital from an outside facility were identified. The clinical and radiographic outcomes were recorded. A cost analysis was performed on patients who were transferred with an isolated, linear, nondisplaced skull fracture.

RESULTS Between 2005 and 2013, a total of 619 pediatric patients with isolated skull fractures were transferred. Of these, $438(70.8 \%)$ patients had a linear, nondisplaced skull fracture. Of these 438 patients, 399 (91.1\%) were transferred by ambulance and $39(8.9 \%)$ by helicopter. Based on the current ambulance and helicopter fees, a total of $\$ 1,834,727$ (an average of $\$ 4188.90$ per patient) was spent on transfer fees alone. No patient required neurosurgical intervention. All patients recovered with symptomatic treatment; no patient suffered late decline or epilepsy.

CONCLUSIONS This study found that nearly $\$ 2$ million was spent solely on transfer fees for 438 pediatric patients with isolated linear skull fractures over a 9-year period. All patients in this study had good clinical outcomes, and none required neurosurgical intervention. Based on these findings, the authors suggest that, in the absence of abuse, most children with isolated, linear, nondisplaced skull fractures do not require transfer to a Level 1 pediatric trauma center. The authors suggest ideas for further study to refine the protocols for determining which patients require transport.

http://thejns.org/doi/abs/10.3171/2015.9.PEDS15352

KEY WORDS pediatric; linear; skull fracture; transfer; seizures; vomiting; cost; trauma

$\mathcal{C}$ HILDREN diagnosed with skull fractures are often transferred to a hospital with pediatric neurosurgical capabilities, even if the child has no underlying brain injury. Prior studies have demonstrated a number of potential complications that may arise in patients with skull fractures, including seizures, intractable vomiting, decline in neurological status, and cerebral hemorrhage necessitating neurosurgical intervention. Currently, an estimated $85 \%$ of pediatric patients diagnosed with isolated skull fractures are admitted to a hospital. ${ }^{2,8}$ The decision to admit a patient is based on the presumed risk of decline that requires specialty intervention. However, this risk is estimated based on data compiled before advanced imaging technology was widely available. More recent reviews show low rates of neurological decline and medical intervention in patients with isolated skull fractures with no associated clinical or radiographic risk factors. ${ }^{8}$

Modern CT scanning is now readily available even in small emergency departments. Telemedicine is also becoming more common, allowing the transmission of radiographic images to specialists at a tertiary care center for consultation. 
The financial cost of health care is under increasing scrutiny in the United States. Expensive practices must be reevaluated. Practices that maximize outpatient care are encouraged because the cost of admitting a patient to the hospital is 3 to 4 times greater than the cost of treating and discharging the patient from the emergency department. ${ }^{1,4-8}$ Transport costs are high and add to the financial burden.

In this study, we analyze the transfer costs of patients with isolated skull fractures who were transported to a Level 1 pediatric trauma center between 2005 and 2013. We also evaluate the clinical and radiographic outcomes of these patients. Our goal is to provide data to help guide recommendations that are both clinically logical and fiscally responsible.

\section{Methods}

The study began after receiving approval from the Indiana University Institutional Review Board. We retrospectively evaluated the medical and radiographic records of all patients admitted between 2005 and 2013 for skull fractures to Riley Hospital for Children (Indianapolis, Indiana), a Level 1 pediatric trauma institution. Patients were identified by the ICD-9 skull fracture codes. We excluded patients with intracranial hematoma or intraparenchymal injury. We also excluded patients with known or highly suspected multisystem injuries.

Patients who were transferred from an outside hospital to Riley Hospital for Children were identified. The transfer costs were calculated based on the local rates of the major providers of air and ambulance transport. All transport services included distance in their algorithms for cost; thus, the distances traveled were calculated for each transferred child. The decision to transfer a patient rests primarily with the outside hospital working with $\mathrm{Ri}$ ley Hospital's transportation center. The neurosurgeon at Riley is often notified or consulted. The mode of transfer (helicopter vs ambulance) is based on the evaluation of the clinical urgency by the outside emergency physician. Historically, Riley accepts all requested trauma transfers.

\section{Recorded Demographic Information}

Patient age at admission, hospital length of stay, detailed mechanism of injury, location of fracture, Glasgow Coma Scale score noted by the transferring hospital, Glasgow Coma Scale score upon arrival to Riley Hospital, and the mode of transfer (ambulance or helicopter) were recorded. Clinical symptoms were recorded, including intractable vomiting, seizures, and other injuries not identified on the initial evaluation. The types of medical treatments that were administered were identified, such as intracranial pressure monitoring, external ventricular drain placement, and other surgical interventions. Follow-up data from the neurosurgical service or the patient's pediatrician at a minimum of 1 year after injury were analyzed. Any subsequent imaging studies were noted.

\section{Results}

\section{Mechanisms and Location of Injuries}

A total of 1426 patients were treated for traumatic brain injuries over the 9-year study period (Table 1). Of these 1426 patients, $724(50.8 \%)$ patients had isolated skull fractures with no parenchymal injuries, hematomas, or other concurrent injuries. The mechanism of injury was most commonly falls or blunt trauma (74.3\%), followed by nonaccidental trauma (NAT) (7.3\%), bike or skateboard accidents $(6.1 \%)$, motor vehicle accidents (5.5\%), all-terrain vehicle or moped accidents (3.9\%), and a pedestrian being struck by vehicle accidents (2.9\%). The most common anatomical location of the fracture was the parietal bone (312 patients; 39.2\%), followed by the occipital bone (27.9\%), frontal bone (18.8\%), and temporal bone (14.1\%). A few patients sustained fractures that extended into several bones, and 2 patients had fractures extending into the skull base.

Of the 724 patients with isolated skull fractures, 619 $(85.5 \%)$ were transferred from an outside hospital by either helicopter or ambulance. There were 181 patients who were transferred with depressed skull fractures. The remaining 438 patients were transferred with linear, nondisplaced skull fractures.

\section{Patients Transferred With Linear, Nondisplaced Skull Fractures}

The demographic data, mechanisms of injury, and anatomical locations of the skull fractures are shown in Table 2 . The average patient age in the linear fracture group was $3.4 \pm 4.1$ years, which was similar to that of the depressed fracture group $(3.9 \pm 4.1$ years). There was a higher percentage of males than females (63.5\% vs $36.5 \%)$. The mechanisms of injury and anatomical locations of the fractures were similarly distributed as the entire treated population.

\section{Calculation of Transfer Costs}

The transfer costs of the 438 patients with linear, non-

TABLE 1. Mechanism of injury and location of all patients treated for isolated skull fractures during the study period

\begin{tabular}{cc}
\hline Variable & No. of Fractures $(\%)$ \\
\hline Total isolated skull fractures & 724 \\
\hline Mechanism of injury & $538(74.3)$ \\
\hline Falls/blunt trauma & $44(6.1)$ \\
\hline Bike/skateboard & $40(5.5)$ \\
\hline Motor vehicle accident & $53(7.3)$ \\
\hline NAT & $21(2.9)$ \\
\hline Pedestrian & $28(3.9)$ \\
\hline All-terrain vehicle/moped & \\
\hline Anatomical location & 796 \\
\hline Total fractures* & $112(14.1)$ \\
\hline Temporal & $150(18.8)$ \\
\hline Frontal & $312(39.2)$ \\
\hline Parietal & $222(27.9)$ \\
\hline Occipital &
\end{tabular}

* The number of fractures exceeds the number of patients because certain patients had multiple fractures, and fractures affecting multiple bones were counted at each anatomical location. 
TABLE 2. Demographic data, mode of transport, and cost analysis of 438 patients transferred with isolated, linear, nondisplaced skull fractures

\begin{tabular}{|c|c|}
\hline Variable & Value $^{*}$ \\
\hline Mean age \pm SD (yrs) & $3.4 \pm 4.1$ \\
\hline \multicolumn{2}{|l|}{ Sex } \\
\hline Male & $278(63.5)$ \\
\hline Female & $160(36.5)$ \\
\hline \multicolumn{2}{|l|}{ Mode of transfer } \\
\hline Ambulance & $399(91.1)$ \\
\hline Air & $39(8.9)$ \\
\hline \multicolumn{2}{|l|}{ Mechanism of injury } \\
\hline Falls/blunt trauma & $335(76.5)$ \\
\hline Bike/skateboard & $30(6.8)$ \\
\hline Motor vehicle accident & $20(4.6)$ \\
\hline NAT & $31(7.1)$ \\
\hline Pedestrian & $9(2.0)$ \\
\hline All-terrain vehicle/moped & $13(3.0)$ \\
\hline \multicolumn{2}{|l|}{ Anatomical location } \\
\hline Total fractures & 478 \\
\hline Temporal & $67(14.1)$ \\
\hline Frontal & $80(16.7)$ \\
\hline Parietal & $185(38.7)$ \\
\hline Occipital & $146(30.5)$ \\
\hline \multicolumn{2}{|l|}{ Transfer costs } \\
\hline$\leq 2$ yrs old & $267(\$ 1,112,127)$ \\
\hline Ambulance & $244(\$ 846,070)$ \\
\hline Air & $23(\$ 266,087)$ \\
\hline NAT & $31(\$ 107,492.50)$ \\
\hline Ambulance & $31(\$ 107,492.50)$ \\
\hline Air & $0(\$ 0.00)$ \\
\hline$>2$ yrs old & $171(\$ 722,566.50)$ \\
\hline Ambulance & $155(\$ 537,462.50)$ \\
\hline Air & $16(\$ 185,104)$ \\
\hline NAT & $0(\$ 0.00)$ \\
\hline Total & $438(\$ 1,834,727.40)$ \\
\hline Ambulance & $399(\$ 1,383,532.50)$ \\
\hline Air & $39(\$ 451,194.90)$ \\
\hline
\end{tabular}

* Values are reported as the number of fractures (\%) unless otherwise indicated. Transfer costs are reported as the number of transfers (cost).

displaced skull fractures are shown in Table 2. Most of the patients (399 patients; 91.1\%) were transferred by ambulance, and $39(8.9 \%)$ patients were transported by helicopter. The average distance for ambulance transfers was 126.7 miles, and the distance for helicopter transfers was 131.2 miles. Ambulance transfer fees were based on the distance traveled, with a $\$ 300$ base fee and $\$ 25$ per mile thereafter. Helicopter fees were based on time in the air and calculated individually by the carriers.

The average ambulance transfer fee was $\$ 3467.50$. Thus, the estimated cost for the ambulance transfers of 399 patients was $\$ 1,383,533$. The average helicopter trans- fer cost was $\$ 11,569$; thus, the estimated cost of the 39 helicopter transfers was $\$ 451,195$. The total transfer costs for 438 pediatric patients with isolated linear skull fractures were estimated to be $\$ 1,834,727$, with an average cost of $\$ 4188.90$ per patient.

\section{Patients Younger Than 2 Years}

Of the 438 patients, 267 (61.0\%) patients were children younger than 2 years. Most were transferred by ambulance (244 of 267 patients; 91.4\%). Twenty-three (8.6\%) were transported by helicopter. The total transfer costs of the children younger than 2 years of age were $\$ 1,112,157$.

Thirty-one (11.6\%) of these patients were identified as victims of abuse or NAT. There was no diagnosis of NAT in any child older than 2 years.

\section{Clinical Outcomes}

Among the 438 patients transferred for linear, nondisplaced skull fractures, none experienced neurological decline or required neurosurgical intervention (Table 3). Only $9(2.1 \%)$ patients had experienced episodes of intractable vomiting that required intravenous fluid support. Eighteen of these $438(4.1 \%)$ patients required treatment from another medical service for injuries that were not identified on the initial outside evaluation.

Peri-injury seizures were documented in 9 of the total 619 patients (1.4\%) transferred with either a linear or depressed skull fracture. Six of these were patients with only linear fractures (1\% of total; $1.4 \%$ of linear fracture patients). All patients with seizures were younger than 2 years, except one 12-year-old child who suffered a skull fracture from a fall that was caused by a seizure. All seizures were witnessed before or during the child's initial presentation at the initial transferring hospital. There was no definite association between seizures and either the anatomical location of the fracture or the mechanism of injury. All patients who experienced seizures were started on seizure treatment at the initial hospital, and there were no episodes of status epilepticus or late seizures.

\section{Clinical and Radiological Follow-Up Data}

The clinical and radiological outcomes were evaluated

TABLE 3. Clinical outcomes of 438 transferred patients with isolated, linear, nondisplaced skull fractures

\begin{tabular}{lc}
\hline \multicolumn{1}{c}{ Variable } & No. $(\%)$ \\
\hline Neurological decline & 0 \\
\hline Neurosurgical intervention & $0(0)$ \\
\hline Intracranial pressure monitoring & $0(0)$ \\
\hline External ventricular drain placement & $0(0)$ \\
\hline Craniotomy & $0(0)$ \\
\hline Bur hole & $0(0)$ \\
\hline Elective neurosurgical intervention & $0(0)$ \\
\hline Intractable vomiting & $9(2.1)$ \\
\hline Seizure & $6(1.4)$ \\
\hline Treatment for nonneurosurgical injuries not identified on & $18(4.1)$ \\
the initial examination & \\
\hline
\end{tabular}


for all 619 patients transferred for skull fractures. Of these, $42(6.8 \%)$ patients received additional CT scanning while in the hospital; none of these patients had any new clinically relevant findings. Outpatient follow-up CT scanning was performed on $560(90.4 \%)$ patients. There were no clinically relevant additional findings noted on follow-up imaging. Clinical follow-up data were available at 1 year for $90 \%$ of the patients. These patients were followed by either their pediatrician or the neurosurgical service. No new long-term clinical symptoms or late seizures were identified.

\section{Discussion}

It is a common practice to transfer children with isolated skull fractures to a pediatric trauma center because of the potential for decline that may require specialized intervention. Studies have demonstrated a number of potential complications that must be considered in patients with skull fractures, including seizures, intractable vomiting, decline in neurological status, and cerebral hemorrhage necessitating neurosurgical intervention. ${ }^{2,3,6-9}$ However, these potential complications are based on prior studies in the literature, many of which preceded the current widespread availability of CT scanning.

Over the 9-year study period, 619 patients were transferred to Riley Hospital for Children (a Level 1 pediatric trauma center) with isolated skull fractures. No patients required subsequent neurosurgical intervention. Nine patients had peri-injury seizures, including 1 patient whose seizure preceded the fall that caused the fracture. No patients developed epilepsy.

Of the total patient population, 438 transferred patients had linear, nondisplaced skull fractures. Nine of these $438(2.1 \%)$ patients had prolonged vomiting that required intravenous fluid hydration and medications. Eighteen (4.1\%) patients were treated for injuries that were not identified at the primary evaluation prior to transfer. NAT was the mechanism of injury in $31(7.1 \%)$ patients. This rate is consistent with the findings of Laskey et al. ${ }^{4}$ All of the patients with NAT were younger than 2 years. The transfer costs for these 31 patients was $\$ 107,493$. Excluding the patients with NAT, the total transfer costs of the patients with linear, nondisplaced skull fractures was estimated to be $\$ 1,727,234.50$.

A second or follow-up CT scan was obtained for 560 of the 619 transferred patients (90.4\%). Previously at our institution, CT scanning was performed routinely during the outpatient follow-up appointment. No new clinically relevant findings were discovered. Follow-up clinical data were available at 1 year for $90 \%$ of patients. No patients had new long-term neurological findings or epilepsy.

\section{Financial Considerations}

The financial concerns in the current medical environment in the United States are forcing clinicians to reevaluate accepted practices. Our data suggest a low rate of complications that require specialty care in pediatric patients who have sustained isolated linear, nondisplaced skull fractures. In our series, nearly $\$ 2$ million was spent just on the transfer of these children over a 9-year period.
Moreover, the cost of admitting these patients is 3 to 4 times higher than the cost of observing and discharging a patient to home. This represents a discrepancy of $\$ 1,500$ to $\$ 2,000$ per patient at our institution and is consistent with the literature. ${ }^{2,5,6}$ Transferred patients are universally admitted for observation at our institution. Thus, the cost of admission could also be added to every patient transferred. In total, we calculated that more than \$3.5 million was spent purely on the transfer and admission of patients with a very low risk of need for neurosurgical intervention. This estimated cost is before the addition of any treatment costs (intravenous fluid administration, laboratory work, imaging, etc.).

\section{Limitations}

The study is limited by its retrospective nature. We also did not have access to the original records of the transferring facility. Thus, our data are based solely on the records of the receiving hospital (Riley Hospital). Therefore, mitigating circumstances leading to the decision to transfer may have been missed. The costs were estimated, although we made every effort to make this as accurate and representative as possible.

\section{Conclusions}

Our data suggest an extremely low risk of clinical decline that requires neurosurgical intervention in patients with linear, nondisplaced skull fractures. There was a low rate of prolonged vomiting that was treated with standard therapy. No patients suffered late seizures. A small percentage of patients $(4.1 \%)$ required treatment for nonneurosurgical injuries that were not identified on the initial examination. There were no new, neurosurgically relevant findings on follow-up head CT scan. The cost of transfer alone was estimated to be $\$ 1,834,727$ over the 9-year study period for children with linear, nondisplaced skull fractures without other injuries. Moreover, the actual time from evaluation at the outside facility to arrival via transfer to the tertiary care center was often well over 6 to 8 hours, which is the suggested observation time for these patients. Thus, many patients likely could have been safely discharged from the original facility rather than transferred to a Level 1 pediatric trauma center. $2,8,9$

The possibility of abuse must be considered in children with head injury. In our series, 31 of the 438 (7.1\%) patients with linear, nondisplaced skull fractures were victims of NAT. All of these patients were younger than 2 years. Unfortunately, we do not have data on how many of these patients were suspected of abuse at the initial evaluation versus those whose abuse was discovered later. It is currently our policy to have a social evaluation for all children younger than 2 years with a skull fracture. We believe that any child with even a slight suspicion of NAT should be transferred to a center with dedicated child abuse services available, especially if they are younger than 2 years.

A follow-up CT scan was obtained for $90.4 \%$ of patients, either in the hospital or in an outpatient follow-up setting. No new, clinically relevant findings were discovered. There is a growing concern about the long-term effect of ionizing radiation on children. We have subsequent- 
ly reevaluated the practice of "routine follow-up CT." We now recommend follow-up imaging in these patients only when clinically indicated (e.g., neurological decline and concern for spinal fluid leakage).

We therefore suggest that most children with isolated skull fractures do not need transfer to a Level 1 center. Most patients require observation and symptomatic support that is well within the capabilities of most emergency departments. However, for various reasons, community physicians may not feel comfortable observing headinjured children, even if the risk of decline is low. The growing availability of telemedicine may help identify patients who truly need transfer to a tertiary care facility. Pediatric neurosurgeons are now often able to remotely view locally acquired $\mathrm{CT}$ images. Thus, a specialist may be able to consult with a local emergency department to identify radiographic risk factors such as fractures near a major vascular structure, fracture depression, pneumocephalus, or intracranial blood. The neurosurgeon may be able to counsel the treating physician in signs of abuse. It is important to note that we excluded patients with many of the characteristic radiological findings of abuse, such as a subdural hematoma or concurrent injuries (rib or longbone fractures). A consulting neurosurgeon may be able to detect subtle but worrisome radiographic signs of NAT and recommend transportation to an appropriate facility.

We encourage neurosurgeons to develop national and local protocols for transfer. We encourage efforts to increase the ease and availability of telemedicine and remote specialty consulting. With educational efforts and collaboration with local facilities, we believe many children may avoid a costly, often unnecessary transfer.

\section{References}

1. Erlichman DB, Blumfield E, Rajpathak S, Weiss A: Association between linear skull fractures and intracranial hemorrhage in children with minor head trauma. Pediatr Radiol 40:1375-1379, 2010

2. Greenes DS, Schutzman SA: Infants with isolated skull fracture: what are their clinical characteristics, and do they require hospitalization? Ann Emerg Med 30:253-259, 1997

3. Gruskin KD, Schutzman SA: Head trauma in children younger than 2 years: are there predictors for complications? Arch Pediatr Adolesc Med 153:15-20, 1999

4. Laskey AL, Stump TE, Hicks RA, Smith JL: Yield of skeletal surveys in children $\leq 18$ months of age presenting with isolated skull fractures. J Pediatr 162:86-89, 2013

5. Mannix R, Monuteaux MC, Schutzman SA, Meehan WP III, Nigrovic LE, Neuman MI: Isolated skull fractures: trends in management in US pediatric emergency departments. Ann Emerg Med 62:327-331, 2013

6. Metzger RR, Smith J, Wells M, Eldridge L, Holsti M, Scaife ER, et al: Impact of newly adopted guidelines for management of children with isolated skull fracture. J Pediatr Surg 49:1856-1860, 2014

7. Reid SR, Liu M, Ortega HW: Nondepressed linear skull fractures in children younger than 2 years: is computed tomography always necessary? Clin Pediatr (Phila) 51:745-749, 2012

8. Rollins MD, Barnhart DC, Greenberg RA, Scaife ER, Holsti M, Meyers RL, et al: Neurologically intact children with an isolated skull fracture may be safely discharged after brief observation. J Pediatr Surg 46:1342-1346, 2011

9. Russo CA, Owens PL, Stocks C: Common injuries that result in hospitalization, 2004: Statistical Brief \#19. Healthcare Cost and Utilization Project (HCUP) Statistical Briefs. Rockville, MD: Agency for Health Care Policy and Research, 2006

\section{Disclosures}

The authors report no conflict of interest concerning the materials or methods used in this study or the findings specified in this paper.

\section{Author Contributions}

Conception and design: all authors. Acquisition of data: all authors. Analysis and interpretation of data: all authors. Drafting the article: all authors. Critically revising the article: all authors. Reviewed submitted version of manuscript: all authors. Approved the final version of the manuscript on behalf of all authors: Fulkerson. Study supervision: Fulkerson.

\section{Correspondence}

Daniel H. Fulkerson, Goodman Campbell Brain and Spine, Riley Hospital for Children, 702 Barnhill Dr. \#1134, Indianapolis, IN 46202-5200. email: dfulkers@iupui.edu. 